\title{
Outlier Robust Draper and Lin Designs
}

\author{
Ahmed F. Siddiqi \\ School of Business \& Economics \\ University of Management \& Technology \\ Pakistan \\ ahmedfsiddiqi@gmail.com
}

\begin{abstract}
Design robustness to a single outlier is studied for Draper 1990 designs by introducing and applying minimax outlier effect criterion. The criterion uses outlying effect of design points and attempts to minimizes the maximum outlying effect for a design point to have design with almost equal outlying effects and the design is adjusted for maximum outlying effect. Resultant outlier robust Draper \& Lin designs have been exhibited as more compact, so less resource intensive, as compare to the other existing equivalent designs.
\end{abstract}

Keywords: Draper \& Lin designs, Minimax Effect Criterion, Outlier, Outlier Robust Designs, Robust Designs.

\section{Introduction}

Standard central composite designs (CCD), developed by Box \& Wilson (1951), for fitting second-order models usually have a large number of design points, especially when the number of factors under consideration is large (Angelopoulosa \& Koukouvinosa 2008) These CCD by their very construction, are quite huge and thus resource intensives (see Montgomery 2001 for details on the construction and other characteristics of CCD). And these huge volumes become an ominous deterrent in their practical applications (Chen, Lin \& Zhang 2003, Bulutoglu \& Cheng 2003,amomg many) It is always desirable to reduce the number of runs as much as possible while maintaining the ability to estimate all of the terms in the proposed model. Draper (1985) and Draper (1990)(DL) composed small response surface designs by reducing the number of factorial points in standard CCD. These reduced factorial points are extracted, usually and mostly, from the age-old Plackett \& Burman (1946) designs by intelligently selecting some of its columns and rows. These designs are quite popular for their cherished characteristics especially in the domain of pharmacy. Numerous examples in this regard are found in academic literature like Gracia, et all (2003) who use the efficiency of DL designs in optimizing both operational and chemical crucial variables affecting a FIA-chemiluminescence detection system. Gracia, et all (2003a) use these designs in determination of albumin in biological fluids by flow injection analysis using the peroxyoxalate chemiluminescent system in micellar medium. Santos. Resell \& Collar (2008) use these designs in gelatinization and retrogradation Kinetics of High-Fiber Wheat Flour Blends. An intelligent reduction in size may increase the design's optimality as well, as calculated in terms of alphabetic optimalities (Kiefer \& Wolfowitz 1959). But, this increased optimality is marred by the presence of an outlier. The same Draper \& Lin (DL) designs are studied, here in this paper, in presence of an outlier and it is attempted to develop a DL design that is robust to a single outlier (ORD-1). The 
basic philosophy is taken from Siddiqi (2008) where it is explored in general terms.

Outliers, missing observations, and other related impediments are very common in observational, as well as in compiled data. There are different connotations to this term. Ranging from wrongly recorded to surprising observations, academic literature uses different names for such observations. Beckman \& Cook (1983) and Barnett \& Lewis (1994) have outlined a comprehensive list of such connotations which attempt to define outlying observation with differing vocabularies. However, going by the conceptual similarities exist in the definitions, outliers refers to observations having an exclusive, different, usually vaguely delineated personality of their own. Here, in this paper, this version is adopted as the definition of outliers. Experience shows that in a typical industrial setting 1 to $10 \%$ of all measurements performed for the purpose of the planned experiments results in grossly surprised data and should be treated as outlier (see, for related comments, Daniel (1959), Anscombe (1960), Morgenthaler \& Schumacher (1999)). Such observations erode the balance and sequential conclusioning of an investigation. Whole comparison of the data, and of its interpretation, changes because of these outliers. Most of data analytical technique employs special procedures to tackle them. Quoting Hawkins (1980) and Barnett \& Lewis (1994), the presence of outlier in a set of data may be tackled by

1. their outright rejection from the data by considering it an error,

2. further analyses for these suspected observation to ascertain their veracity,

3. their incorporation by special modifications either in the model or in the design,

4. re-experimentation to be sure what is happening

It has been a common practice among researchers, especially social scientists, to throw these observations out just by labeling them as erroneous. But these cannot be erroneous all the time and in that case the experimenter may run the chance of losing an important, may be the most important, hitherto unknown dimension of the experiment. On the other hand, once the measurements have been taken, it is in most situations impossible to check the validity of single measurements by repeating selected parts of the experiment. It is therefore important to search for specialized methods that may by be used to adjust the design, analysis, and interpretations, for possible surprising observations without kicking them out. Many attempts have been made in this direction. As a matter of fact, most of these attempts are made in the domain of regression analysis which may be labeled as post-design phase of any experiment. While a very little is attempted to control these observations at the pre- or during-design phase. This later philosophy assumes these observations as necessarily correct but surprising and attempts to anticipate them instead of discarding. In other words, this philosophy attempts to develop design robust enough to tackle these observations if there exist any. 
The term robust designs is coined by Box \& Draper (1975) in their attempt to minimize the effect of outliers on the fitted values obtained by the least square estimation based on classified linear model. Their basic philosophy involves the minimization of the variance of discrepancy caused by outliers. They show that the fitted response will be relatively insensitive to outliers if $i^{\text {th }}$ diagonal elements, $h_{i i}$, of the HAT matrix, as it is called in regression literature (Cook \& Weisberg 1982), given by $H=X\left(X^{\prime} X\right)^{-1} X^{\prime}$, are as uniform and identical as possible and suggest $\sum h_{i i}$ as a convenient measure of such uniformity. This uniformity is attained by intelligently choosing axial distance in central composite designs. They have developed designs for a few number of factors and compared these designs with rotatable designs without establishing their superiority. There are other attempts, in the academic literature, focusing exclusively on the development of designs robust to other problems. Draper (1961), Ghosh (1982), Herzberg \& Andrews (1975), Herzberg \& Andrews (1976), Akhter \& Prescott (1986), Akram (1993) are a few names in the list who presented several maneuvering to guard the design against missing observations. Akhter (1985), Akhter \& Prescott (1986), Akram (1993) presented designs based on central composite designs employing a minimax criterion which works on down weighing the effect of a missing observation. There are other problems too for which robust designs exists. Schwabe (1995) studied robustness with respect to the underlying model. Zhou (2001) studied the robust against serially correlated observations. Toman (1992) and Toman \& Gastwirth (1994) uses Bayesian methodology to incorporate the results of the first study (or studies) into the design of the follow up study. Park \& Cho (2003) for example, developed designs robust for outliers and non-normal experimental data. Fellner (1986), Zhou and Zhu (2003) developed outlier robust designs to get more reliable estimates for variance components in random effects models.

This article is an attempt to robustify Draper \& Lin (1990) for possible outliers. Section 2, hereunder, crafts the minimax philosophy for a design, in general, and for a CCD in special. Section 3 shows how this criterion be applied on a DL design to develop a robust version of the design. Section 4 , at the end offer concluding remarks for the article. The appendix, in the last pages, is showing a code written for Mathematica to generate these minimax $\mathrm{DL}$ designs for different configurations of designs points and number of runs.

\section{Minimax Effect Criterion}

In simple vocabulary, the criterion calls for calculation of outlying effects of all design points and then minimizing the maximum outlying effect to have equal outlying effect for all design points thus to adjust the whole design for the maximum outlying effect. The basic idea is developed in Siddiqi (2008), as 
discussed earlier, while here it is been adapted for Draper \& Lin (1990) designs. Adopting usual symbols and notations where a design model assumes the form

$$
\left(\begin{array}{c}
y_{1} \\
y_{2} \\
. \\
y_{n}
\end{array}\right)=\left(\begin{array}{ccccc}
x_{1,1} & x_{1,2} & \ldots & \ldots & x_{1, k} \\
x_{2,1} & x_{2,2} & \ldots & \ldots & x_{2, k} \\
\ldots & \ldots & \ldots & \ldots & \\
x_{n, 1} & x_{n, 2} & \ldots & \ldots & x_{n, k}
\end{array}\right)\left(\begin{array}{c}
\beta_{1} \\
\beta_{2} \\
\cdots \\
\beta_{k}
\end{array}\right)+\left(\begin{array}{c}
\varepsilon_{1} \\
\varepsilon_{2} \\
\ldots \\
\varepsilon_{n}
\end{array}\right)
$$

The outlying effect of a design point may well be given by its relative weight in the whole design, as outliers cause disturbance in the weight structure of the design (Prescott 1975). In alphabetic optimal designs (as derived by Kiefer \& Wolfowitz (1959)), this relative weight of a design point may be evaluated by calculating the relative change in the determinant of the corresponding information matrix, $\left(X^{\prime} X\right)$, by rubbing that design point out (jackknifing, as it is called in literature). If $\left|X^{\prime} X\right|$ denotes the corresponding determinant of the information matrix of an $n$ point design's information matrix for which $j^{\text {th }}$ $(j=1,2, \ldots, n)$ design point is rubbed, the relative change in $\left|X^{\prime} X\right|$, and thus the outlying effect for the $j^{\text {th }}(j=1,2, \ldots, n)$ design point, is given by

$$
O_{j}=\frac{\left|X^{\prime} X\right|-\left|X_{j}^{\prime} X_{j}\right|}{\left|X^{\prime} X\right|}, \text { fo } \mathrm{r} j=1,2, \ldots, n
$$

If $x_{j}$ denotes the $j^{\text {th }}$ design point, then

$$
O_{j}=\frac{\left|X^{\prime} X\right|-\left|X^{\prime} X-x_{j}^{\prime} x_{j}\right|}{\left|X^{\prime} X\right|}, \text { for } j=1,2, \ldots, n
$$

These complex matrices may be translated into simpler matrices due to Rao \& Toutenburg (1995, p.290) as follows,

$$
O_{j}=\frac{\left|X^{\prime} X\right|-\left|X^{\prime} X\right| \cdot\left|1-x\left(X^{\prime} X\right)^{-1} x_{j}^{\prime}\right|}{\left|X^{\prime} X\right|}=x\left(X^{\prime} X\right)^{-1} x_{j}^{\prime}
$$

The resultant expression $x\left(X^{\prime} X\right)^{-1} x_{j}^{\prime}$ happens to be the $(j, j)^{\text {th }}$ diagonal component of the already-known HAT matrix (after Cook \& Weisburg (1982)), given by $H=X\left(X^{\prime} X\right)^{-1} X^{\prime}$. So the outlying effect of $j^{t h}(j=1,2, \ldots n)$ design point is given by $h_{j}$, where $h_{j}$ symbolizes the $j^{\text {th }}$ diagonal component of $\boldsymbol{H}$. Siddiqi (2008) also discussed some interesting characteristic properties of $O_{j}$ defining its behavior under different circumstances. Let us calculate the outlying effects for DL designs to approach an ORD-1. 


\section{Outlier Robust DL Designs}

The data matrix, $\boldsymbol{X}$, for the $\mathrm{DL}$ designs, being the family member of $\mathrm{CCD}$, is composed of three types of points, i.e. factorial, axial and the central points. In the taxonomy laid down by Box \& Wilson (1951), Table 1 gives the factorial points for DL designs with different number of factors. The axial points, two for each factor, in a traditional $2^{k}$ factorial setting, along its axis, are at a distance $\pm \alpha$ from the design center.

\section{Table 1: Design Points for the Draper \& Lin [1990] Designs}

\begin{tabular}{ccl}
\hline$k$ & Runs & Design Points \\
\hline 3 & 4 & I, ab, ac, bc \\
4 & 8 & I, c, ab, ad, bd, abc, acd, bcd \\
5 & 12 & I, d, ab, ae, ce, abc, acd, bce, bcd, bde, acde, abde \\
6 & 16 & $\begin{array}{l}\text { I, be, cd, cf, df, abc, abd, abf, ace, ade, aef, bcde, bcef, bdef, } \\
\text { abcdf, acdef }\end{array}$ \\
& $\ldots$ & $\ldots$ \\
\hline
\end{tabular}

The design does have some points at the center whose number may be determined by using Draper (1982). If $N_{f}, N_{a}$, and $N_{c}$ symbolize respectively the number of factorial, axial, and central points then the total number of design points for a DL design turns out to ben $=N_{f}+N_{a}+N_{c}$. The corresponding HAT matrix, the diagonal elements of which are to be calibrated, would be of an order $(n \times n)$.

The selection of an appropriate axial distance, $\alpha$, is consequential. Wu \& Hamada (2000) opine it to be between 1 and $\sqrt{k}$; for $\alpha=1$ the axial points would be at the center of the faces of the cuboid while for $\alpha=\sqrt{k}$ all the axial and factorial points would be in the same sphere. Composite designs with different desirable properties, like rotatability, robustness, etc., are developed with different selections of $\alpha$. Larger values of $\alpha$ are increasing the efficiency of the designs, in terms of alphabetic optimality, but making them practically more difficult to obtain as they become more resource intensive. In minimax effect criterion, an $\alpha$ is rummaged for which the maximum outlying effect $O_{j}$ $(j=1,2, \ldots, n)$ is minimum.

Going as per, already mentioned, philosophy of minimax designs, the maximum outlying effects are to be calculated for all design points. For a typical CCD, there are three groups of designs points; factorial, axial and central. If $O_{f}, O_{a}$, and $O_{c}$ symbolize respectively the maximum outlying effect for these three groups of points, then, an expression may be developed for these effects in terms of $\alpha$. Unfortunately, there is no general form of such expressions which may be calculated for a typical $2^{k}$ fractional factorial design. This is, mainly, due to nonconstant structure of such designs, especially in case of DL designs, Plackett \& Burman (1946) designs, small CCD developed in Draper (1985), and other such 
derivatives of fractional factorials. These outlying effects happens to be polynomials in varying powers of $\alpha$ and getting very complex with increasing number of factors in the design. However, an exclusive algorithm is developed in Mathematica ver. 7, available in appendix, to compute these expressions for different configurations of DL designs.

For a 3 factor DL design with 2 central points, for example, these are given by

$$
O_{f}=\frac{96-48 \alpha^{2}+11 \alpha^{4}}{12\left(8-4 \alpha^{2}+\alpha^{4}\right)}, O_{a}=\frac{23-12 \alpha^{2}+3 \alpha^{4}}{3\left(8-4 \alpha^{2}+\alpha^{4}\right)}, O_{c}=\frac{6+\alpha^{4}}{6\left(8-4 \alpha^{2}+\alpha^{4}\right)}
$$

Line-graph, in Fig. 1, shows how these outlying effects behave with varying values of $\alpha$, over a range of 0 to 2.5. $O_{f}$ seems to be a decreasing function in $\alpha, O_{a}$ an increasing, while $O_{c}$ seems to be a regular bell shaped curve, at least within this range.

Figure 1: Dynamics of $O_{f}, O_{a}$, and $O_{c}$ with Varying Values of $\alpha$

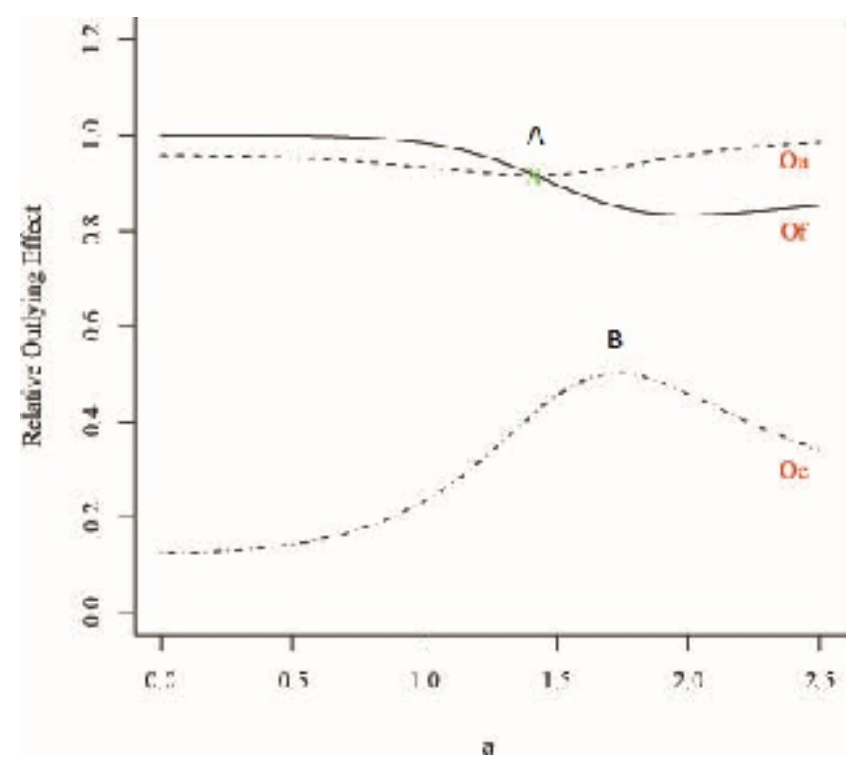

It has been observed that $\sum_{j=1}^{n} O_{j}$ remains the constant for all choices of $\alpha$ (Siddiqi 2008). This makes minimizing $O_{f}$ results in increasing $O_{a}$. However, a point does exist at $\alpha=1.41421$, where $O_{f}=O_{a}$ (see point A in Fig. 1). This is the point at which maximum outlying effects are equal for factorial and axial design points while the maximum outlying effect for central points, $O_{c}$, is quite low (merely 0.41667 observed at point $\mathrm{B}$ in Fig. 1). So, at $\alpha=1.41421$ (point A), the outlying effects of design points are either identical or lesser than that of equioutlying-effect design points and the design is adjusted for this maximum outlying effect. This is the minimax design which is not letting any single design point to desolate whole design by having an unusual value. 
The dynamics of $O_{c}$ is quite interesting to observe. The maxima for the $O_{c}$ decreases with increasing number of center points in the design. Figure 2, in left panel, shows the expressions for $O_{c}$ for different number of center points, as derived by using code available in appendix.

$$
\begin{aligned}
& O_{N_{c}=2}=\frac{\alpha^{4}+6}{6 a^{4}-24 a^{2}+48} \\
& O_{N_{c}=3}=\frac{\alpha^{4}+6}{7 \alpha^{4}-24 \alpha^{2}+54} \\
& O_{N_{c}=4}=\frac{\alpha^{4}+6}{8 \alpha^{4}-24 \alpha^{2}+60} \\
& O_{N_{c}=5}=\frac{\alpha^{4}+6}{9 \alpha^{4}-24 \alpha^{2}+66}
\end{aligned}
$$

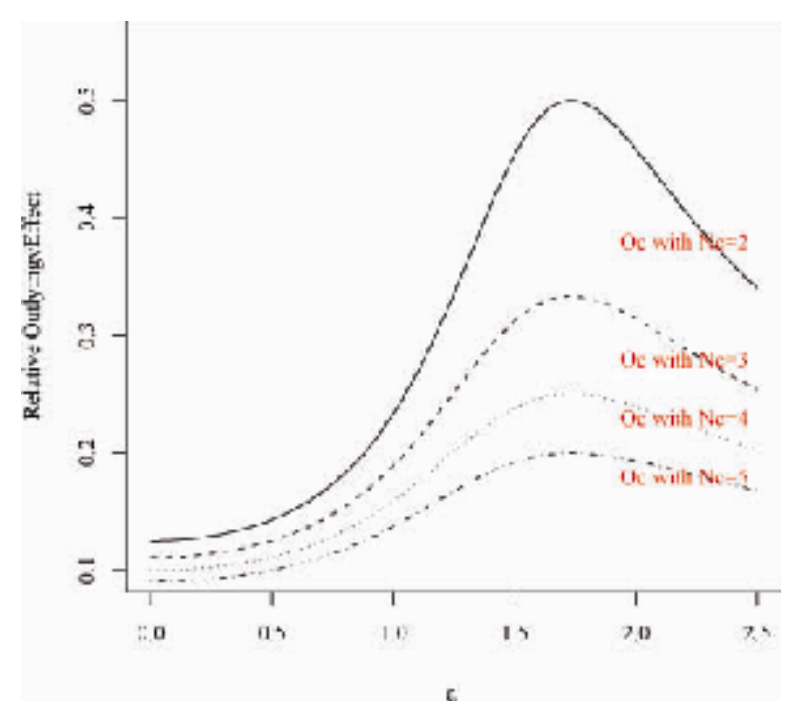

Figure 2: Dynamics of Outlying Effect of Central Points

Its maxima is 0.5 (point B in Fig. 1), and interestingly, this maxima decreases geometrically with increasing $N_{c}$. Figure 2 shows, in the right panel, that this maxima is given by $1 / N_{c}$; i.e. the maxima with two center points is given by $1 / 2=0.5$, with three center points it is $1 / 3=0.333$, and so on. And, this maxima is observed at the same $\alpha$.

A similar exercise may be carried out for other DL designs with varying number of factors. The Mathematica code is available in the appendix which can be used for developing minimax DL designs with other configurations. The code may also be used for varying number of replications in the factorial or axial parts of the design.

These robust $\mathrm{DL}$ designs are comparable with other similar designs like designs with $\alpha=\sqrt{k}$, rotatable designs, Box \& Draper (1975) outlier robust designs. These designs may be compared either on the basis of alphabetic optimality, or with respect to design compactness which results in lesser resource-intensive designs. An optimal design may be more efficient but it may also be resource intensive at the same time. Table 2 shows $\alpha$ for different designs including rotatable, orthogonal, Box \& Draper (1975) (BD) and Minimax designs for a 3 and 4 factor DL design with two and three points at the center respectively along with different alphabetic optimality coefficients for this design. These includes

- A optimality, results in minimizing the average variance of the estimates. So $A$ optimal designs would give minimum possible variances for their parameter estimates, 
- $D$ optimality, which results in maximizing the differential Shannon information content of the parameter estimates. It discusses both $D_{\text {main }}$ and $D_{\text {sqre }}$ which focuses information contents with respect to main effects and squarred terms respectively. So $D_{\operatorname{main}}$ designs would contain maximum information with respect to their main effects, while $D_{\text {sqre }}$ would contains it with respect to squarred terms of the $2^{\text {nd }}$ response surface designs, and

- $E$ optimalities, whic results in minimizing the variance of best linear unbiased estimates (BLUE). So $E$ optimal designs would attempt to create designs with best linear unbiased estimates which have minimum variances.

As a matter of fact, a lesser coefficient for these alphabetic optimalities represents a more efficient design while a lesser $\alpha$ value represents a more compact design. For both of these $\mathrm{DL}$ configurations, minimax designs are more efficient as compare to other compatible designs. In case of 3 factor DL design with two central points, the minimax design yields an $\alpha$ almost equals to that of rotatable designs, greater than that of orthogonal designs but lesser than BD designs. So minimax designs are more compact as compare to BD. Comparing on the basis of alphabetic optimalities, it seems that rotatable designs are almost similar to that of minimax while rest of these competitors are not doing a good job. The minimax designs are not as compact as the others but are giving lesser variances to parameter estimates, and yielding more information. In case of a 4 factor DL design with three central points, the minimax designs are the most compact after the orthogonal but are more optimal in comparison to other given competitors. The differences in these values are small but grows with the size of design. Designs for more factors and with different configuration can be obtained by using the given Mathematica code, in Appendix. The minimax designs are consistently giving better results; as for different design combinations it may either be rotatable, orthogonal, or DL which is better either compactness wise or optimality wise, but the minimax designs always gives an edge on either of these grounds.

As a matter of fact, rotatble and orthogonal designs are kind of generic designs and do not change with the situation at hand to maintain their characteristics. They are not meant for the situations where outliers present. Outliers, missing observations, other related impediments may easily take the cherished properties of these designs away. BD designs are, however, robust in nature and attempt to circumvent these impediments. But these designs, as require variance caused by these impediments, are calculation intensive and for heavy design configuration are almost impossible to develop. Even the Mathematica code given in Appendix, takes double the time to compute BD design as compare to minimax design. 


\section{Table 2: Alphabetic Efficiency Comparison}

For a 3 factor DL Design with two central Points

$\begin{array}{lllll} & \text { Rotatable } & \text { Orthogonal } & \text { BD } & \text { MinMax } \\ \text { alpha } & 1.41421 & 1.21 & 1.698525 & 1.414214 \\ \text { A - optimality } & 3.16667 & 3.81538 & 2.6182 & 3.1667 \\ \text { D- optimality } & 7.94 \times 10^{-8} & 6.58 \times 10^{-7} & 0 \times 10^{-6} & 0 \times 10^{-5} \\ \text { Dmain } & 0.00195312 & 0.00300703 & 0.00107231 & 0.00195313 \\ \text { Dsqre } & 0.00078125 & 0.00334048 & 0.00012598 & 0.00078125 \\ \text { E- optimality } & 0.654508 & 0.83017 & 0.68262 & 0.654508\end{array}$

For a 4 factor $D L$ Design with three central Points

$\begin{array}{lllll} & \text { Rotatable } & \text { Orthogonal } & \text { BD } & \text { MinMax } \\ \text { alpha } & 1.68179 & 1.47119 & 2.029419 & 1.523458 \\ \text { A - optimality } & 2.47509 & 2.87648 & 2.05735 & 2.75634 \\ \text { D- optimality } & 2.27 \times 10^{-15} & 2.61 \times 10^{-14} & 5.44 \times 10^{-17} & 1.39 \times 10^{-14} \\ \text { Dmain } & 0.0000287473 & 0.0000432825 & 0.0000143869 & 0.000039152 \\ \text { Dsqre } & 5.08 \times 10^{-6} & 0.000029389 & 3.89 \times 10^{-7} & 0.000018697 \\ \text { E- optimality } & 0.426777 & 0.532824 & 0.41680 & 0.502246\end{array}$

\section{Concluding Remarks}

The DL designs are widely used especially in pharmaceutical industry for their manageable size, optimality and other properties where these properties are compromised in presence of outliers which are very common in the industrial setup. The paper is an attempt to generate $\mathrm{DL}$ designs which are robust to these outliers. The minimax effect criterion, introduced and used in this paper, yield outlier robust designs for which almost all the design points have equal significance. Such a design would not let any potential outlier, at any design point, to distort the harmony, balance and the sequential conclusioning of the design. Moreover, these designs are more efficient as compare to the existing competitors, e.g. the rotatable, least-outlying-effect-variance Box \& Draper (1975) designs which are making this criterion more useful. Designs robust to a single outlier are introduced in this paper but the idea can easily be generalized for more than a single outlier. However, their are certain limitations are there in these designs like the non-availability of these robust designs for certain design points. For example in the replicated (where factorial parts is replicated) $k=5$ minimax DL designs are not available. 


\section{Ahmed F. Siddiqi}

\section{Appendix}

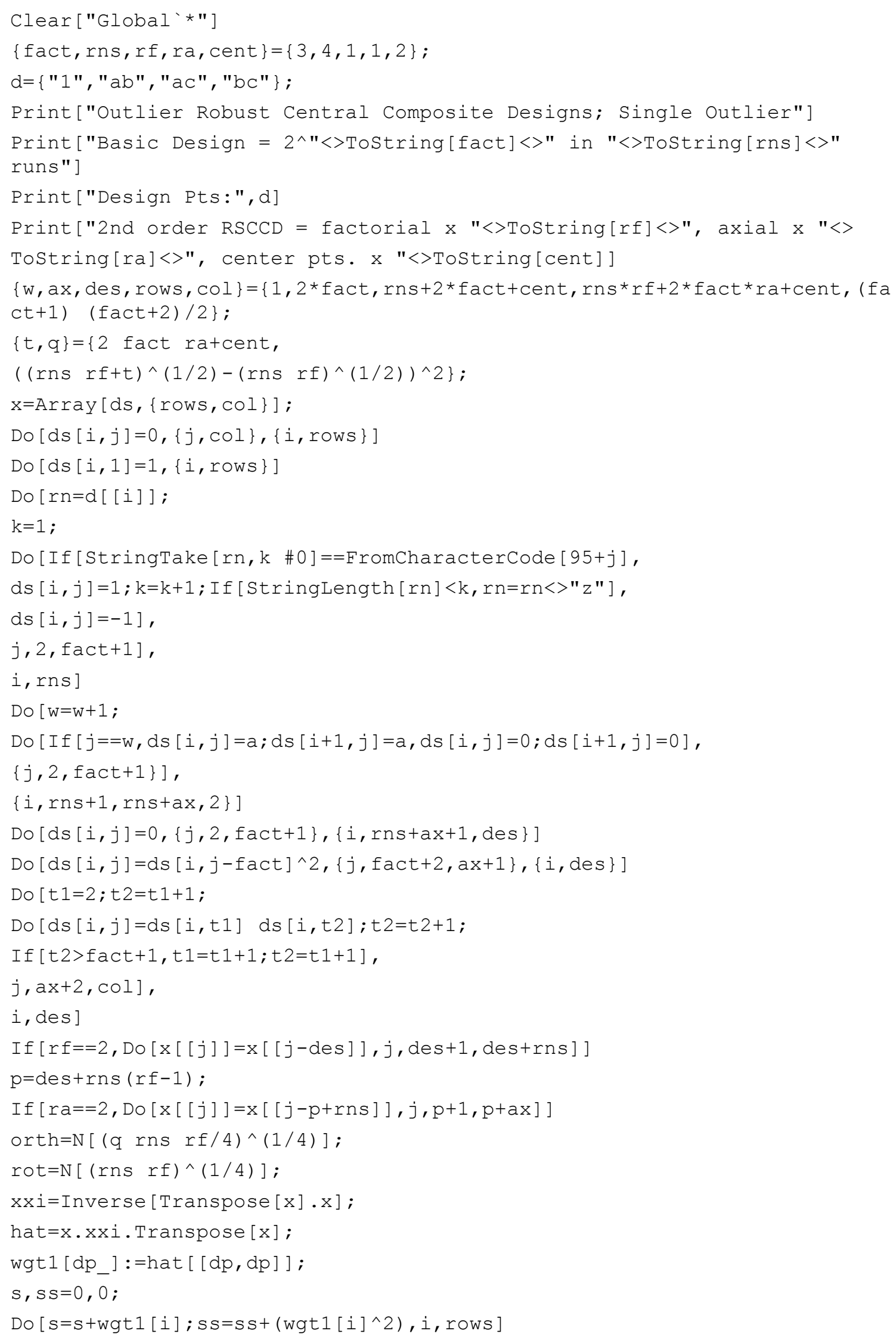




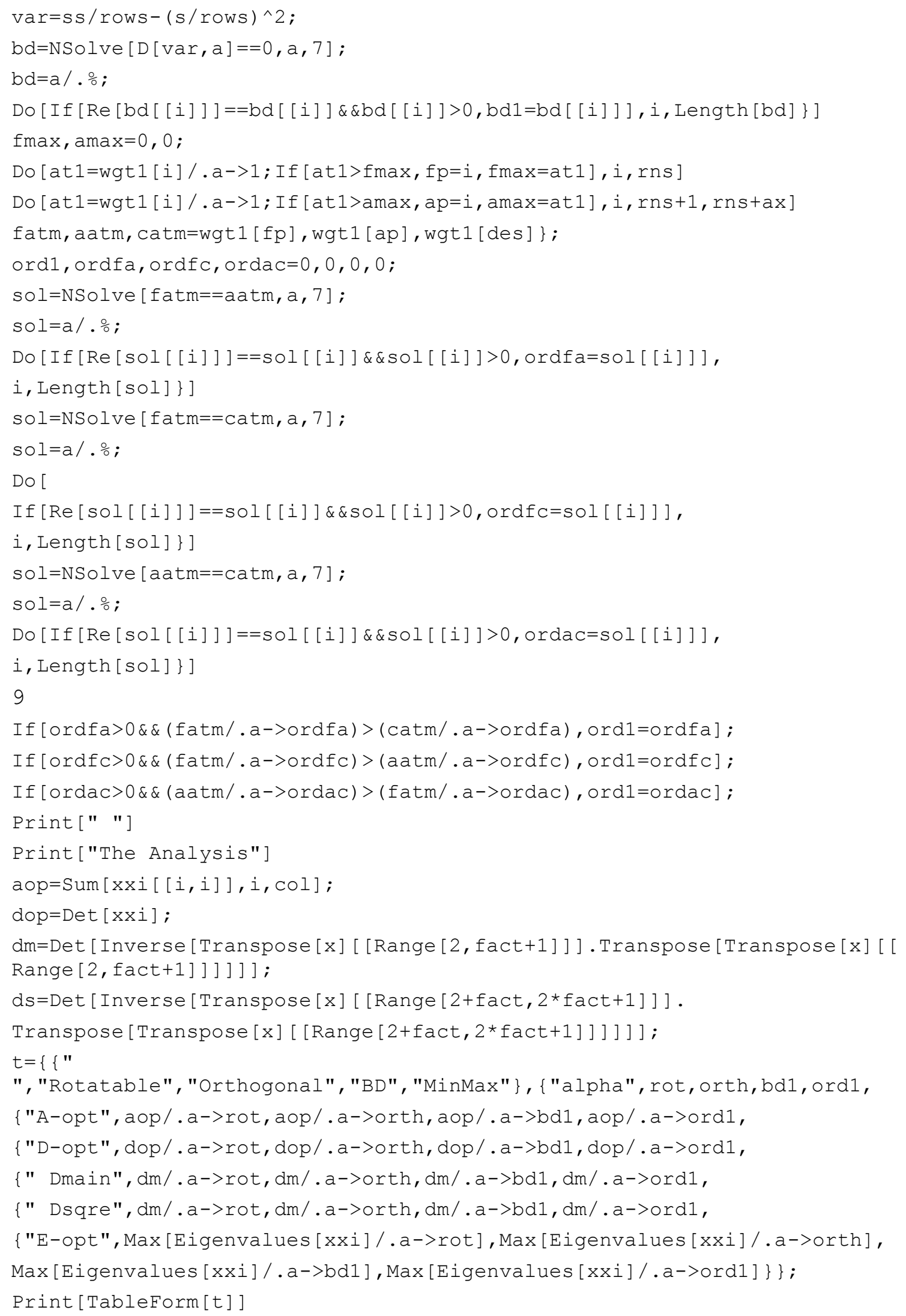




\section{References}

1. Akhter, M. (1985), Response Surface Designs Robust to Missing Observations, PhD thesis, University of Southampton.

2. Akhter, M. \& Prescott, P. (1986), 'Response surface designs robust to missing observations', Communications in Statistics \& Simulations 15(2), 345-363.

3. Akram, M. (1993), Central Composite Designs Robust to Three Missing Observations, $\mathrm{PhD}$ thesis, Islamia University Bahawalpur.

4. Angelopoulosa, P., H. E. \& Koukouvinosa, C. (2008), 'Small, balanced, efficient and near rotatable centralcomposite designs', Journal of Statistical Planning and Inference.

5. Anscombe, F. (1960), 'Rejection of outliers', Technometrics 2, 123-147.

6. Barnett, V. \& Lewis, T. (1994), Outliers in Statistical Data, Wiley, New York.

7. Beckman, R. \& Cook, R. (1983), 'Outliers', Technometrics 25(2), 119-163.

8. Box, G. \& Draper, N. (1975), 'Robust designs', Biometrika 62(2), 347-352.

9. Box, G. \& Wilson, K. (1951), 'On the experimental attainment of optimum conditions', Journal of Royal Statistical Society 13, 1-45.

10. Bulutoglu, D. A. \& Cheng, C.-S. (2003), 'Hidden projection properties of some nonregular fractional factorial designs and their applications', Annals of Statistics 31(3), 1012-1026.

11. Chen, C. L., Lin, R. H. \& Zhang, J. (2003), Genetic algorithms for mdoptimal follow-up designs', Comput. Oper. Res. 30(2), 233-252.

12. Cook, R. \& Weisberg, S. (1982), Residuals \& Influence in Regression, Chapman \& Hall., New York.

13. Daniel, C. (1959), 'Use of half normal plots in interpreting 2 level factorial experiments', Technometrics1, 311-341.

14. Draper, N. (1961), 'Missing values in response surface designs', Technometrics 3(3), 383-398.

15. Draper, N. (1982), 'Center points in second order response surface designs', Technometrics 24(1), 127-133.

16. Draper, N. (1985), 'Small composite designs', Technometrics 27(2), 173-180.

17. Draper, N. \& Lin, D. (1990), 'Small response surface designs', Technometrics 32(2), 187-193.

18. Ghosh, S. (1982), 'Robustness of designs against the uniavailability of data', Indian Journal of Statistics (B) 44(1), 50-62.

19. Gracia, G., Campana, G., Barrero, A. \& Rodriguez, C. (2003), 'Determination of albumin in biological fluids by flow injection analysis using the peroxyoxalate chemiluminescent system in micellar medium', Analytical and bioanalytical chemistry $377(2), 281-286$. 
20. Gracia, G., Rodrguez, C., Almansa-Lopez, Chinchilla, S. \& Campana, G. (2003), 'Use of highly efficient Draper-Lin small composite designs in the formal optimisation of both operational and chemical crucialvariables affecting a fia-chemiluminescence detection system', Talanta 60(2), 523-534.

21. Hawkins, D. (1980), Identification of Outliers, Chapman \& Hall, New York.

22. Herzberg, A. \& Andrews, D. (1975), 'Robust designs of experiments', Bulletin of Institute of Statistics 45(2), 370-374.

23. Herzberg, A. \& Andrews, D. (1976), 'Some considerations in the optimal designs of experiments in non-optimal situations', Journal of Royal Statistical Society (B) 38, 284-289.

24. Kiefer, J. \& Wolfowitz, J. (1959), 'Optimal designs in regression problems', Annals of Mathematical Statistics 32, 271-294.

25. Montgomery, D. (2001), Design \& Analysis of Experiments, 5th edn, Wiley, New York.

26. Morgenthaler, S. \& Schumacher, M. (1999), 'Robust analysis of a response surface design', Chemometrics \& Intelligent Laboratory Systems $47,127-141$.

27. Park, C. \& Cho, B. R. (2003), 'Development of robust design under contaminated and non-normal data', Quality Engineering 15(3), 463-469.

28. Plackett, R. \& Burman, J. (1946), 'The design of optimum multifactor experiments', Biometrika 33, 305-325.

29. Prescott, P. (1975), 'An approximate test for outliers in linear models', Technometrics 17(1), 129-132.

30. Rao, C. \& Toutenburg, H. (1995), Linear Models; Least Squares \& Applications, Springer, New York.

31. Santos, E., Resell, C. M. \& Collar, C. (2008), 'Gelatinization and retrogradation kinetics of high-fiber wheat flour blends: A calorimetric approach', Cereal Chemistry 85(4), 455-463.

32. Siddiqi, A. (2008), 'Outlier robust designs', International Journal of Applied Mathematics and Statistics 13(1).

33. Toman, B. (1992), 'Bayesian robust experimental designs for the one-way analysis of variance', Statistics\& Probability Letters 15(5), 395-400.

34. Toman, B. \& Gastwirth, J. L. (1994), 'Efficiency robust experimental designs and estimation using a databased prior', Statistica Sinica 4, 603-615.

35. Wu, C. \& Hamada, M. (2000), Experiments; Planning, Analysis, and Parameter Design Optimization, Wiley Series in Probability \& Statistics, Wiley, New York. 